\title{
An Information Model for Landscape Management - Discussing Temporality Aspects
}

\author{
Sabine Buckl, Alexander Ernst, Florian Matthes, and Christian M. Schweda \\ Technische Universität München, Institute for Informatics, \\ Boltzmannstr. 3, 85748 Garching, Germany \\ \{buckls, ernst, matthes, schweda\}@in.tum.de \\ http://www. systemcartography.info
}

\begin{abstract}
Planning, managing, and maintaining the evolution of the application landscape is a focal point of enterprise architecture (EA) management. Whereas, planning the evolution of business support provided by the business applications is understood as one challenge to be addressed in landscape management, another challenge arises in the context of traceability of management decisions.

This paper discusses the requirements regarding support for landscape management as risen by practitioners from industry, gathered in an extensive survey during which the tool support for EA management was analyzed. Thereby, a lack of support for this management discipline was discovered, which is caused by the way, application landscapes are modeled in tools. We subsequently discuss how to incorporate these requirements into an information model.
\end{abstract}

Keywords: EA management, modeling, temporality, historization.

\section{Motivation and Introduction}

Over the last years enterprise architecture (EA) management has become an important management area, many companies are currently executing or planning to introduce in the nearby future. As a consequence, a multitude of methods for EA management has been developed by academic communities (cf. [1 2 3] ), standardization bodies (cf. 4]), or practitioners (cf. 513]). Although these methods differ substantially concerning the quantity, abstractness, and granularity of the EA documentation, needed for EA management, the need for a documentation is common. Thus, different methods and models for creating such a documentation as well as for maintaining its timeliness have been subjected to research, commonly attributing this documentation as a model of the EA (cf. [6]).

The methods and models developed have to address different challenges arising in the context of EA management, especially when the management of the application landscap 11 is concerned. During information gathering not only information about the as-is situation of the landscape has to be collected, but also

${ }^{1}$ The term application landscape refers to the entirety of the business applications and their relationships to other elements, e.g. business processes in a company. We do not use the term application portfolio, which we regard to have a narrower focus.

G. Feuerlicht and W. Lamersdorf (Eds.): ICSOC 2008, LNCS 5472, pp. 363-374, 2009.

(C) Springer-Verlag Berlin Heidelberg 2009 
information about future aspects, e.g. projects changing the application landscape, has to be maintained. In order to get an overview on the relationships and dependencies of the various elements of the enterprise, different kinds of visualizations, which we refer to as software maps, are typically used (see e.g. V-30 in [1]). Different versions of visualizations are commonly used to illustrate the evolution of the application landscape, either the status quo or future business support. In order to create these documentations, the respective data has to be stored in a repository corresponding to an information model, which defines the elements and the moment in time the information is related to (planned for).

Furthermore, landscape management is closely connected to project portfolio management, as the selected project portfolio determines the future development of the application landscape. Regarding the state of the art in the context of project portfolio management, most decisions about portfolios are currently based on gut feel, not on information, which is derived from a comparison of different variants of the landscape regarding quantitative or qualitative aspects (cf. [7]). The landscape variants therein should be related to the project portfolios, they result from. These variants have to be stored to facilitate comparisons and therefore be used to provide decision support.

EA management follows a typical management cycle consisting of the phases: Plan - Do - Check - Act (cf. [89]). Thereby, the traceability 2 of management decisions taken in the Plan phase and implemented in the Do phase, must be ensured to control the achievement of objectives (Check). An exemplary question in this context could be: Is the status of the planned landscape reached within the planned time frame or has the plan been changed? This information is subsequently used to prepare the next management cycle (Act). Consequently, a third type of information has to be stored in an information model for landscape management besides the planned for and the variant information: the moment in time the landscape was modeled (modeled at). From this discussion the following research question has been derived:

How should an information model for landscape management be designed to incorporate both business and technical aspects, and to support future planning and traceability of management decisions?

This question takes aspects of temporality as connected to landscape management into account. Therein, different versions of the landscape are of importance: the current, planned, and target version. The current landscape represents the status quo of the landscape as is. The planned landscape represents a future state of the landscape as to be at a specific time in the future 3 . This state is modeled by an architect at a certain time, emphasizing e.g. the changes performed by projects up to that specific future date. As a long term perspective the target landscape shows the architecture of the application landscape as envisioned at

\footnotetext{
$\overline{2}$ Traceability of decisions can be achieved by storing previous states of the managed objects. The respective technique is mostly referred to as historization.

${ }^{3}$ In some publications on EA management(cf. e.g. [13]), the terms as-is and to-be are used for the respective landscape version. We do not use this terminology, as the term to-be is often used ambiguously for both planned and target landscapes.
} 
a certain time. Thereby, there is no need to have projects defined transforming the current or planned landscape into the target one. Furthermore, the target landscape does not necessarily specify deployed business applications but refers to envisioned future support providers.

Summarizing, the traceability aspects of landscape management lead to three different time-related dimensions: Firstly, a landscape is planned for a specific time, secondly, a landscape has been modeled at a certain time, and thirdly, different variants of a planned landscape may exist. Figure 1illustrates the relationships between current, planned, and target landscape as well as the different dimensions relevant for landscape management.

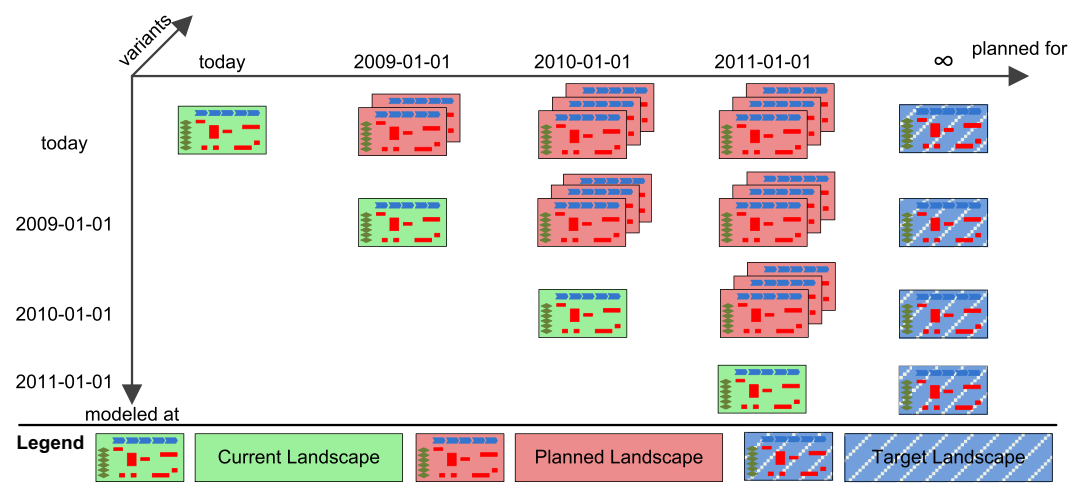

Fig. 1. Current, planned, and target landscape

The research question is approached in this article as follows: Section 2 gives an overview on current approaches to landscape management as described by researchers and practitioners in this field. Further, requirements - especially time-related ones - for an information model for landscape management are introduced. Thereby, a framework for the analysis of the support for landscape management is established. Alongside this framework an analysis of the current tool support for landscape management is performed in Section 3 Section 4 discusses ideas, which could be used to create an information model for landscape management fulfilling the aforementioned requirements. Therein, especially ideas originating from related modeling disciplines are considered. Finally, Section 5] hints at further areas of research in the context of EA management.

\section{Requirements for and Current Approaches to Landscape Management}

Due to the importance of managing the application landscape as a constituent of EA management, a number of different ways to approach this task have been 
proposed both in practice and academia. Subsequently, we give an overview on these approaches with an emphasis on the aspect of temporality.

In [10] the application landscape is referred to as a concept specifying the enterprise's business applications and their interdependencies. This information is reflected in the information model of [10] via interfaces utilized to interconnect the applications. References from these application level concepts (on the application layer as in the notion of [10]) to business level entities, e.g. the different types of business processes (on the organizational layer of the model), are present and can describe the way, how business support is provided. The question at which organizational unit which business process is supported, by which business application, cannot be answered based on the information model. The aspect of temporality is also only partially addressed, while the models contain ways to store life cycle states of applications, it does neither support planning transitions between life cycle states nor does it take projects into account.

In [11 the business applications ands their relationships to other EA constituents form an important information asset, which should be presented to managers to provide decision support. As presentation form of choice, they introduce a type of visualizations, called landscape maps, in which the business applications are related to business functions and products. This relationship is referred to in 11] as a ternary one, which could also be established between applications and two other concepts, although such considerations are not detailed in the article. Temporal aspects are not part of the approach, while ways to use the landscape map visualizations for interacting and changing the data in the underlying models are explicitly stated.

A slightly different focus on managing the application landscape is taken in 12. Therein, the aspect of the interfaces connecting the business applications is analyzed. The number of interfaces associated to a business application is considered an important impact factor, e.g. when changes to the application landscape are considered. In this context, [12] puts special emphasis on documenting and analyzing the current application landscape. This information is used as input to coordinate potential change processes affecting the landscape. While 12 takes a rather detailed look on the business applications and their interconnections, relationships to business related concepts of the EA are not presented in the paper. Whereas, the topic of the evolution of the application landscape is indicated, actual planning of future states or transformation projects is not discussed.

Beside the academic community also practitioners address the field of landscape management. In [5] the overall architecture of the application landscape is considered an important topic of EA management, exerting strong influence on the overall success of the company. Detailing the aspects of landscape management, 5] emphasizes on the relationships of the applications to the business processes, they support, as well as to logical structuring principles, e.g. organizational units. Further, the importance of application landscape planning is referred to, by complementing the current landscape with a target landscape, 
not solely consisting of business applications but also of more abstract providers of support for business processes. Issues of how to transform from the current to the target landscape are also discussed in [5], although concept of planned landscapes is not introduced. Further topics, e.g. traceability of management decisions, are not considered therein.

In [13] the application landscape is presented as a management subject embedded in the context of business and technical concepts, ranging from business processes to technical platform modules. The current landscape should, accordingly, be documented with references to these aspects, especially the technical ones. Complementing the current landscape, a so called ideal landscapt 4 should be defined as part of a landscape management endeavor, incorporating technical visions of the landscape. Mediating between current and ideal, different to-be landscapes 5 should be developed,of which each is assigned to a set of projects, which must be executed to realize the to-be landscape. Here, a strong relationship between the projects and the to-be landscapes is maintained, nevertheless means for tracing back the evolution of a to-be landscape are not incorporated.

Subsuming the state-of-the-art in managing application landscapes as presented in literature, different approaches are employed especially concerning the aspect of temporality. Nevertheless, creating an information model of the application landscape is a widely accepted prerequisite employed in landscape management. In some of the papers, presented above, information models are provided. These information models differ widely regarding the concepts introduced and the relationships as well as regarding their complexity. We regard, notwithstanding, such a model to be mandatory to approach landscape management as a whole and the important aspect of temporality in special.

Due to great interest from industry partners in information about EA management tools and especially their capabilities to address the concerns arising in the context of landscape management, an extensive survey - the Enterprise Architecture Management Tool Survey 2008 - was conducted [14. The survey was developed in cooperation with 30 industry partners. The survey pursued a threefold evaluation approach, relying on two distinct sets of scenarios together with an online questionnaire. Thereby, the first set of scenarios focuses on specific functionality, an EA management tool should provide, without connecting these functionalities to the execution of a typical EA management task, e.g. 1) flexibility of the information model or 2) creating visualizations,. The EA management tools are further evaluated by the scenarios of the second set, which reflect tasks that have been identified as essential constituents of many EA management endeavors, e.g. 1) business object management, or 2) SOA transformation management. One of the most prominent scenarios of the second part is the scenario landscape management, which is concerned with the managed evolution of the application landscape [15. The concern of the scenario was described by the industry partners as follows:

\footnotetext{
${ }^{4}$ Target landscape in the terms used throughout this paper.

${ }^{5}$ In this paper, these landscape are called planned ones.
} 
Information about the application landscape should be stored in a tool. Starting with the information about the current landscape potential development variants should be modeled. The information about the current application landscape and future states should be historicized to enable comparisons. 14.

Subsequently, a catalog of typical questions in the context of landscape management as raised by the industry partners is given:

- What does the current application landscape look like today? Which business applications support which business process at which organizational unit?

- How is, according to the current plan, the application landscape going to look like in January 2010? Which future support providers support which business process at which organizational unit?

- What was, according to the plan of 01-01-2008, the application landscape going to look like in January 2010?

- How does the target application landscape do look like?

- What are the differences between the planned landscape according to the plan of 01-01-2008 and the current plan?

- What projects have to be initiated in order to change from the planned landscape (according to the current plan) to the target landscape? What planning scenarios can be envisioned and how do they look like?

Based on the questions from the industry partners and the different dimensions relevant for landscape management, the following requirements regarding an information model can be derived, the model thus must:

(R1) contain a ternary relationship in order to support analyzes regarding current and future business support (which business processes are supported by which business applications at which organizational units),

(R2) provide the possibility to specify envisioned business support providers in order to facilitate target landscape planning without having to specify implementation details of the business support,

(R3) support the deduction of future landscapes from the project tasks, which execute the transition from the current to the future business support,

(R4) foster the creation of landscape variance based on distinct project portfolios in order to tightly integrate project portfolio management activities, and

(R5) ensure the traceability of management decisions by storing historic information of past planning states. This information may be interesting especially if complemented with information on the rationale for the decisions.

Based on these requirements, an overview about the support for landscape management as provided in the approaches from literature is given in Table 1.

\section{Tool Support for Landscape Management}

The solutions of nine major players in the market of EA management tools were analyzed regarding the information models, which they come shipped with. Three different exemplary approaches as taken by the different tools are subsequently explicated to provide an overview about the current operationalizations 
of landscape management. The attributes are thereby not shown to improve readability but are mentioned in the description, if necessary for understanding.

Prior to discussing the different approaches taken by the tools, the core concepts of landscape management, which are most likely to be represented as classes in the information models, are briefly introduced (for details see [1]):

BusinessProcess: A business process can, according to Krcmar [16], be defined as a sequence of logical individual functions with connections between them. The process here should not be identified with single process steps or individual functions, but with high-level processes at a level similar to the one used in value chains. Thus, a process can have a predecessor process and a successor process, expressed by a respective relationship.

DeployedBusinessApplication: A deployed business application is a software system, which is part of an information system in an organization. The term refers to an actual deployment. In landscape management, business applications are restricted to applications that support at least one process.

FutureSupportProvider: A future support provider poses a sort of envisioned planning object, to be used instead of an actual deployed business application in a target landscape to define a business support.

OrganizationalUnit: An organizational unit represents a subdivision of the organization according to its internal structure, e. g. the entities showing up in an organigram can be used as organizational units.

Project: Adaptations of the application landscape are performed by projects, which each hold different attributes with temporal information, e.g. for their startDate and endDate6. Additionally, a project is plannedAt and removedAt referring to the planning time of its creation and of its deletion - effectively resulting in a time interval of validity, which is assigned to each project. A relationship between the project and the concepts affected by it, e.g. deployed business applications, exists.

SupportProvider: A support provider is an abstract concept, representing an entity, which can provide support for a business process at a specific organizational unit. In the context of the information model, actually deployed application systems can be used as SupportProvider instances as can future business applications.

SupportRelationship: This concept represents the support of a specific business process by a specific support provider at a specific organizational unit.

Starting with a basic approach to landscape management tool 1 presents an information model containing landscape management related concepts, as shown in Figure 2. Here, the business process is connected with the organizational unit via the support provider to support target landscape planning (cf. R2). Whereas data gathered according to this information model can support basic analyzes of the business support for a business process, the relationship to the organizational unit, where the support takes place, is not derivable unambiguously (cf. R1).

${ }^{6}$ If more detailed modeling of projects should be performed, the temporal information could be extended to contain starting and ending dates for different phases of the project, e.g. planning and development. 


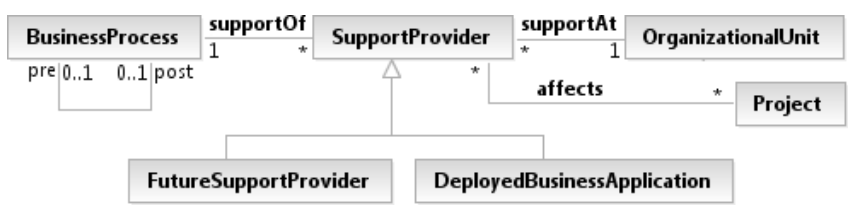

Fig. 2. Information model of tool 1

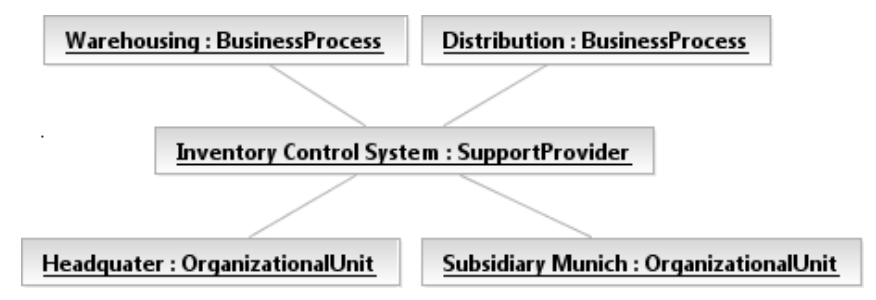

Fig. 3. Instance data corresponding to information model of tool 1

Figure 3 shows exemplary data instantiating the information model from Figure 2, Analyzing this data, a statement, which business process is supported by the Inventory Control System at the Subsidiary Munich cannot be made.

Besides the missing ternary relationship between business process, organizational unit, and support provider, the only concept carrying temporal information - the project - is connected to the support provider via the relationship affects. Thus, no time information for the business support provided can be stored (cf. R3). In addition, planning variants of the landscape can only be built based on the support providers instead of the business support provided (cf. R4). Consequently, tool 1 only rudimentarily supports the management of current, planned, and target landscapes. While such information might be sufficient for future planning in a one dimensional manner, the requirements concerning traceability and versioning cannot be addressed (cf. R5).

The information model of tool 2 (see Figure 4) incorporates the ternary relationship between the business processes, the organizational units, and the support providers by introducing a dedicated class and respective associations (cf. R1). The association supportBy is further assigned life cycle parameters using a mechanism similar to an association class in UML. Thus, it is possible to indicate that the business support provided by a specific instance of class SupportProvider is at a certain point in time in a specific life cycle phase, e.g. planned or active (cf. R2). This notion of life cycle is nevertheless disconnected from the concept of the project, which is independently associated to the class realizing the ternary relationship. While this association allows to model, that the support for a specific business process executed at a specific location is affected by a project, no mechanism to indicate, which SupportProvider actually is changed by the project, is present (cf.R3 and R4)7. Further, the model does

\footnotetext{
7 This fact is caused by the $*$ multiplicity on the SupportProvider end of the supportBy association.
} 


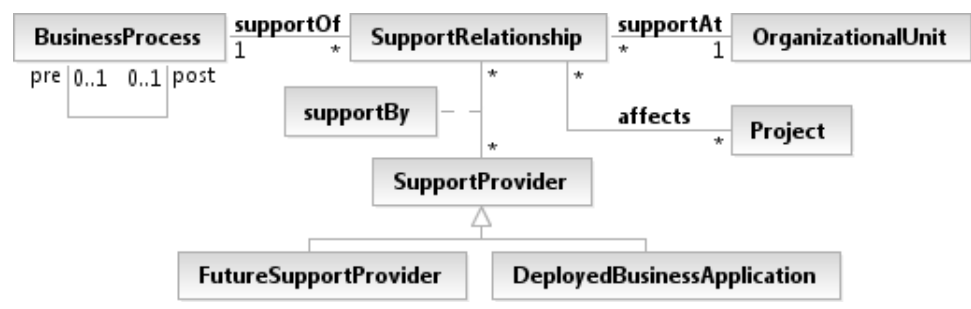

Fig. 4. Information model of tool 2

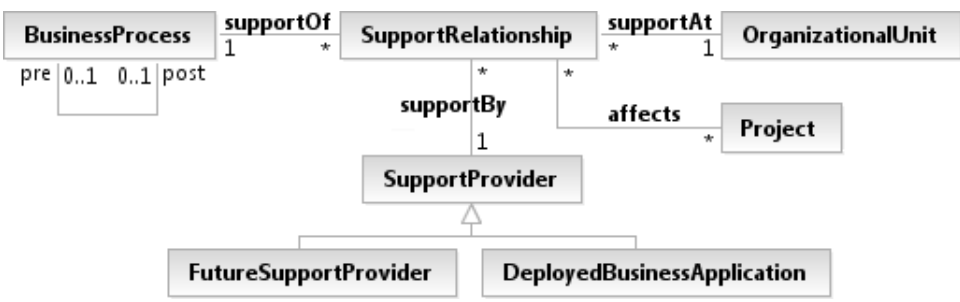

Fig. 5. Information model of tool 3

not support the creation of different landscape scenarios, as it is not possible to make projects or providers of business support belong together in one scenario. A mechanism for marking a SupportProvider an element of a target landscape is nevertheless provided via a flag attribute target in the association class supportBy. Historization of planned application landscapes is not supported (cf. R5) as no means for versioning instances corresponding to the model are given.

Finally, the information model of tool 3 is presented (cf. Figure [5), which is only slightly different from the model of tool 2, provides additional support for application landscape management - future state considerations are supported similarly as in tool 2 (cf. R2). The information model contains a support relationship, which supports analyses regarding the business support provided for a business process by a business application at an organizational unit (cf. R1). Nevertheless, the information model as proposed by tool 3 also implements temporality in a one dimensional manner by the project concept (cf. R3 and R4), which affects the support relationship and contains temporal information, e.g. start and end dates. Such information might be sufficient for planning the evolution of the EA, but is somewhat limited concerning traceability of changes to the plans (cf. R5), which would demand support for bitemporal modeling. As an example, one might think of a plan for the EA regarding the year 2010, which might look different as-of begin 2008 respectively begin 2009 .

Table 1 provides an overview about the evaluation results of the tool support for landscape management. Thereby, the support provided by the different approaches is indicated by symbols ranging from complete fulfillment of the requirement (O) via partial fulfillment ( ) to approaches, which totally lack support for the analyzed requirement $(\bigcirc)$. 
Table 1. Existing Approaches and Tools and their Fulfillment of the Requirements

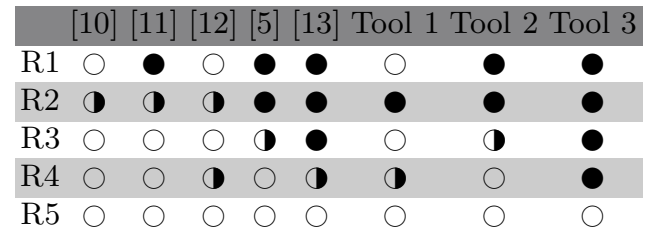

In order to discuss the constituents of an information model fulfilling the requirements, we subsequently detail on related modeling techniques.

\section{Discussing an Information Model for Landscape Management}

The question, how to incorporate aspects of time in a database system has been repeatedly discussed in scientific literature (see e.g. 17[18]). A simple approach is to introduce a time stamp attribute to the table, which should be enriched with temporal information. This allows to specify that an entry of the table is valid since the point in time specified by the time stamp. The approach has the disadvantage that it is not possible to specify that the information stored in the table row is valid for a certain period of time. In order to resolve this problem another attribute can be introduced to define up to which point in time the values are valid, thereby capturing the history of a changing reality.

If traceability should also be considered a so called transaction time has to be specified additionally to the valid time, which has been described before. According to [18] this can be done by introducing two additional attributes defining a time interval for capturing the sequence of states of a changing table. Such a table is than called a bitemporal table.

Similar discussions have taken place for object-oriented models. From these discussions, a few design patterns, of which [19] gives a good overview, have emerged. In addition, 19 introduces basic time concepts: event, time interval, and duration, of which the latter ones can be considered of special importance for our design issue, e.g. for modeling life cycle information of business applications.

Additionally useful in the context of creating temporality aware object-oriented models are the temporal (design) patterns presented in [20]. The concept of the temporal association introduced there can be utilized to model, that the objects referenced in association can change over time. The actual realization of the pattern introduces a class with a validity interval making the association explicit. Other design patterns for addressing temporality exist, e.g. the edition [20], but are not further discussed here.

In order to fulfill the requirements as mentioned in Section 2, especially R4 and R5, which have not been well addressed by the majority of tools, temporal patterns, as alluded to above, could be utilized. This challenge can be met as a central relationship of the landscape management models - the ternary one relating support providers, business processes, and organizational units, which is 
explicated as an independent class, the SupportRelationship. Thereby, the pattern of the temporal association [20] could be incorporated - the associated projects could help to supply periods of validity for the SupportRelationship instances, i.e. the referenced SupportRelationship becomes valid, once the endDate of the project is reached.

If landscape plans for the same point in time created at different times should be compared to each other (cf. R5), the information concerning the point, when the project has been planned at, had to be considered. Consistently, the temporal pattern edition (cf. [20]) could be used to implemented this mechanism.

\section{Outlook}

In this article, we discussed time-related issues of application landscape management and how they relate to other tasks in EA management, especially project portfolio management. Section 2 showed different approaches to landscape management as found in literature. Further, we discussed requirements for landscape management, as gathered from EA management practitioners during an extensive survey, and finally compared the findings from literature and practice. Section 3 discussed the tool support for landscape management with an emphasis on the underlying information models. From this, the drawbacks of the different approaches were explained. Related fields of modeling were taken into account in Section 4 discussing, how an information model could be created to fulfill the requirements. Therein, especially temporal patterns for object oriented models proved to be promising.

Two interesting directions of research result in continuation of the discussions undertaken in this paper. At first, the ideas for constructing a temporal information model for landscape management have yet not been validated. This would nevertheless be an important step. Thereby, the creation of such a model is likely to comprise additional difficulties, especially if considered in the context of a larger EA information model. Second, a temporal information model is due to its inherent complexity likely to be usable only via an appropriate tool. This is especially true considering the temporal relationships to be maintained by a user - constrastingly, currently no tool capable of managing such an information model in a convenient way exists.

The other research direction emphasizes that landscape management is not the only part of EA management that has strong related issues associated. Managing the infrastructure of the enterprise might be also influenced by different dimensions of time. Temporal aspects addressed in one part of EA management may also exert certain influences to other parts of managing the EA, which independently might not be affected by temporality aspects. In order to address this, the information model could be organized in patterns, which keep temporality related issues contained in one fragment of a larger model. A technique potentially helpful in this context is the EA management pattern approach [1]. 


\section{References}

1. Buckl, S., Ernst, A., Lankes, J., and Matthes, F.: Enterprise Architecture Management Pattern Catalog (Version 1.0, February 2008). Technical Report TB 0801, Chair for Informatics 19, Technische Universität München (2008)

2. Lankhorst, M., et al.: Enterprise Architecture at Work - Modelling, Communication, and Analysis. Springer, Heidelberg (2005)

3. Winter, R., Bucher, T., Fischer, R., Kurpjuweit, S.: Analysis and Application Scenarios of Enterprise Architecture - An Exploratory Study. Journal of Enterprise Architecture 33, 33-43 (2007)

4. The Open Group: The Open Group Architectural Framework Version 8.1.1 (cited 2008-10-03), http://www.opengroup.org/architecture/togaf8-doc/arch/

5. Dern, G.: Management von IT Architekturen (Edition CIO). Vieweg, Wiesbaden (2006)

6. Fischer, R., Aier, S., Winter, R.: Enterprise Modelling and Information Systems Architectures. In: Proceedings of the 2nd International Workshop EMISA 2007, St. Goar, Rhine (2007)

7. Lankes, J., Schweda, C.: Using Metrics to Evaluate Failure Propagation in Application Landscapes. In: Multikonferenz Wirtschaftsinformatik (MKWI) 2008, München (2008)

8. Deming, W.: Out of the Crisis. Massachusetts Institute of Technology, Cambridge (1982)

9. Shewart, W.: Statistical Method from the Viewpoint of Quality Control. Dover Publication, New York (1986)

10. Braun, C., Winter, R.: A Comprehensive Enterprise Architecture Metamodel and Its Implementation Using a Metamodeling Platform. In: Desel, J., Frank, U. (eds.) Gesellschaft für Informatik 2005, Bonn (2005)

11. van der Torre, L., Lankhorst, M., ter Doest, H., Campschroer, J., Arbab, F.: Landscape Maps for Enterprise Architectures. In: Dubois, E., Pohl, K. (eds.) CAiSE 2006. LNCS, vol. 4001, pp. 351-366. Springer, Heidelberg (2006)

12. Garg, A., Kazman, R., Chen, H.-M.: Interface descriptions for enterprise architecture. Science of Computer Programming 61, 4-15 (2006)

13. Engels, G., Hess, A., Humm, B., Juwig, O., Lohmann, M., Richter, J.-P., Voss, M., Willkomm, J.: Quasar Enterprise - Anwendungslandschaften serviceorientiert gestalten. dpunkt Verlag, Heidelberg (2008)

14. Matthes, F., Buckl, S., Leitel, J., Schweda, C.: Enterprise Architecture Management Tool Survey 2008. TU München, Chair for Informatics 19 (sebis), Munich (2008)

15. Aier, S., Schönherr, M.: Enterprise Application Integration - Flexibilisierung komplexer Unternehmensarchitekturen. Gito, Berlin (2007)

16. Krcmar, H.: Informations management, 4th edn. Springer, Berlin (2005)

17. Date, C.: An Introduction to Database Systems. Addison Wesley, Boston (2000)

18. Snodgrass, R.: Developing Time-Oriented Database Applications in SQL. Morgan Kaufmann Publishers, San Francisco (2000)

19. Anderson, F.: A Collection of History Patterns. In: Harrison, N., Foote, B., Rohnert, H. (eds.) Pattern Languages of Program Design. Addison Wesley, Boston (1999)

20. Carlson, A., Estepp, S., Fowler, M.: Temporal Patterns. In: Harrison, N., Foote, B., Rohnert, H. (eds.) Pattern Languages of Program Design. Addison Wesley, Boston (1999) 